\title{
EXPERT AE SIGNAL ARRIVAL DETECTION
}

\author{
Milan Chlada \\ Institute of Thermomechanics AS CR, Czech Republic
}

\begin{abstract}
Accurate acoustic emission (AE) source location is the primary goal of the defect analysis following the AE signal detection. The source localization is mostly based on arrival time differences of signals recorded by several transducers. Considerable signal distortion happens during the wave propagation through the solid. Inaccurate determination of signal onset and arrival time differences respectively, are the greatest sources of localization errors. Especially, in a case of higher requirements on accuracy and robustness, the results of currently used localization methods appear to be insufficient. In the paper, recently improved version of the new signal - shape based algorithm, modeling an expert system of the elastic wave arrival detection, is introduced. In many applications, this method, based on signal energy and local gravity center evolution, has been proved as rugged enough, fast and easily applicable.
\end{abstract}

\section{KEYWORDS}

Acoustic emission, signal arrival detection.

\section{Introduction}

In present, there exist various approaches to the AE signal arrival detection [1] such as e.g. the threshold crossing method, the first signal onset detection, time differences detection by means of cross-correlation function, and the methods outgoing from the wavelet transformation, or detecting local statistical parameters changes of the signal in terms of time series [2]. However, with respect to accuracy, automation and robustness, these methods often fail to satisfy more exacting requirements resulting from the large and variant $A E$ signal sets processing needs. $A$ number of algorithms also demand to resize input parameters even more times within one measurement data analysis (e.g. due to the different channels characteristics of measuring device). 


\section{IV ${ }^{\text {rd }}$ NDT in PROGRESS}

For the fast and rugged signal processing needs, the new signal edge detection algorithm was proposed. The method should reach relevant results for AE signals of various complex shapes, different noise levels, partially broken, highly distorted or incomplete (see fig.1). The algorithm goal is to simulate an expert ("visual") elastic wave arrival time detection based on local gravity center or signal energy evolution.
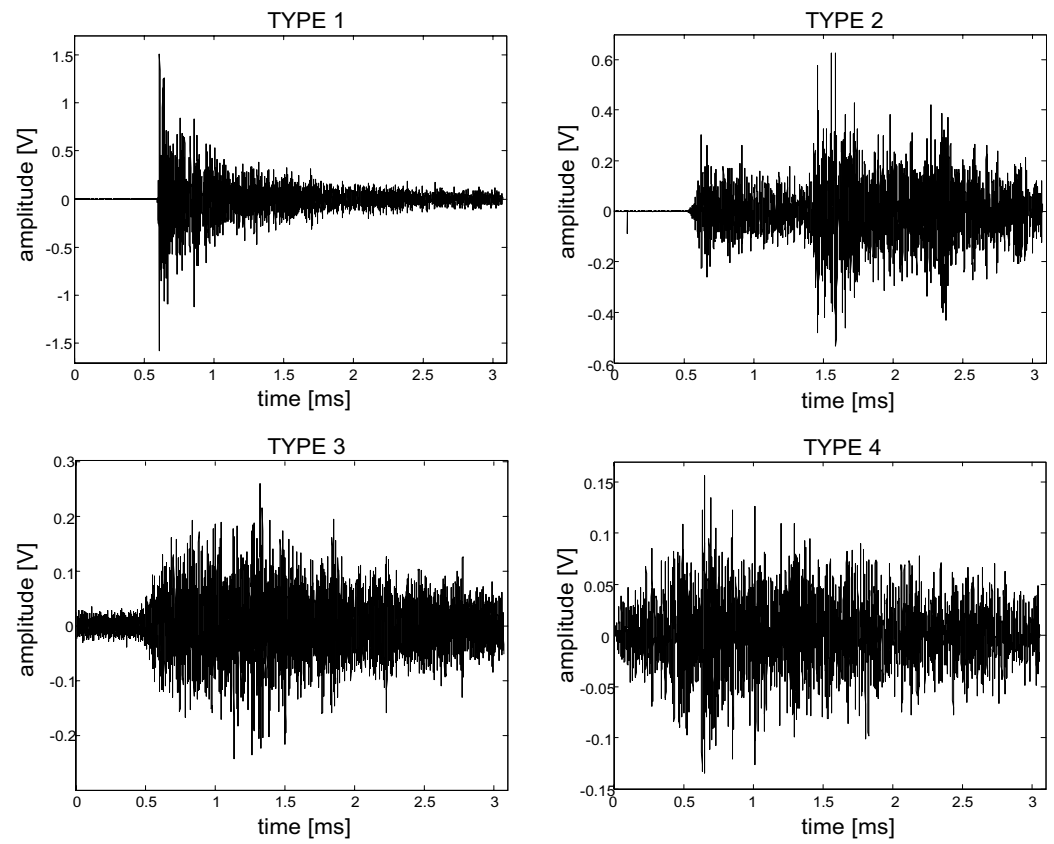

Fig.1: typical AE signal shapes

\section{Algorithm description}

\subsection{Measuring window length computation}

The unique algorithm input is sampled signal $s=\{s(j) \mid j=1, \ldots, N\}$. First of all, the window length adjusting parameter $k$ is computed. To suppress the local gravity center (2.5) vector oscillation, maximum window width $(=2 k+1)$ should slightly exceed the "main" data record frequency wave length, i.e. double maximum length between the neighbouring signal mean value crossings. The measure of "exceeding" is represented by parameter $c_{k}$, which should not be, indeed, too extensive. Unlikely, the method accuracy would decrease. Parameter $k$ is computed as follows:

$$
k=c_{k} \cdot \max (\operatorname{diff}(z))
$$

Where the auxiliary vector $\boldsymbol{Z}=\left\{\boldsymbol{Z}(m) \mid m=1, \ldots, N_{m}\right\}$ is made up by $N_{m}$ ascendantly sorted signal sample indexes, after which zero crossing occures (when the signal is already centered):

$\boldsymbol{z}(m)=j_{m}$ pro $\forall m: 1 \leqslant m \leqslant N_{m} \wedge\left|d\left(j_{m}\right)\right|=2 \wedge 1 \leqslant j_{m}<j_{m+1}<N$, where $d=\operatorname{diff}(\operatorname{sign}(s))$ 
The difference operation is defined as distinction between original vector samples:

$$
\operatorname{diff}(x)=\{x(j+1)-x(j) \mid j=1, \ldots, N-1\}
$$

\subsection{Auxiliary local gravity center vector}

Subsequently, for all signal samples, the auxiliary vector $g$ is computed. It's $\mathrm{i}$ - th element is the gravity center y-coordinate $C G_{Y}\left(\left|s_{i}\right|\right)$ of existing absolute signal part $s_{i}$ around the relevant sample $i$ :

$$
g(i)=G C_{Y}\left(\left|s_{i}\right|\right)=\frac{\sum_{j=1}^{k_{i}}\left(k_{i}+1-j\right)\left|s_{i}(j)\right|}{\frac{1}{2}\left(k_{i}^{2}+k_{i}\right)}
$$

Where above mentioned existing signal part is considered as:

$$
s_{i}=\{s(j) \mid j=\max \{i-k, 1\}, \ldots, \min \{i+k, N\}\}
$$

\subsection{Automatic noise separation and approximate signal edge appointment}

The key problem of designed automatic algorithm is an approximate signal noise part separation. Considering signal samples as the unique input data, it is needed to propose special functions providing distinct amplitude increase detection and, afterwards, noise pretrigger removal. Due to dispersion and various reflections, the steepest signal rise many times doesn't precede maximum amplitude. Further, one data record can represent more overlapping signals and, therefore, maximum amplitude doesn't need to belong to first event, that is preferred (see fig.1 - type2).

Let's assume that the initial record samples are realization of a random process with constant mean value and variance. Unlike the threshold detection, the new algorithm should not respond to solitary amplitude deviations as e.g. electrical interferences or $A D$ converter failures (see fig. 1 - type2). In practice, we often meet situations when the signal beginning is captured just very closely by the first record samples or actually anywise. Automatic algorithm should solve even those situations and by means of mathematically modeled expert knowledge detect signal noise part absence or the event record incompleteness. In terms of above mentioned requirements, following functions $q_{1}$ and $q_{2}$ were established:

$$
q_{1}(i)= \begin{cases}0 & i=1 \\ \frac{(i-1)(g(i)+g(1))}{\sum_{j=2}^{i}(g(j-1)+g(j))} & \text { for } i=2, \ldots, N\end{cases}
$$

Function $q_{1}$ compares the surface below the join of the first and i-th local gravity centers vector sample with the sum of surfaces below each sample joins in analogical interval (see fig.2). This ratio detects the first marked gravity centers auxiliary vector rise and, therefore, also significant rise of the signal itself. 
Vice - versa, it doesn't reflect unique signal deviations. Relative algorithm stability is provided in this way,. The peak sample index $m_{1}$ of vector $q_{1}$ determines the first distinct rise point of the signal .

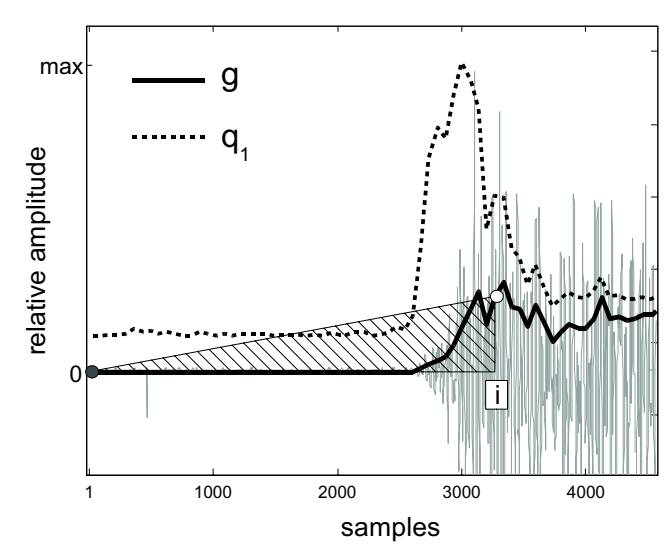

Fig.2: vector $q_{1}$ computing

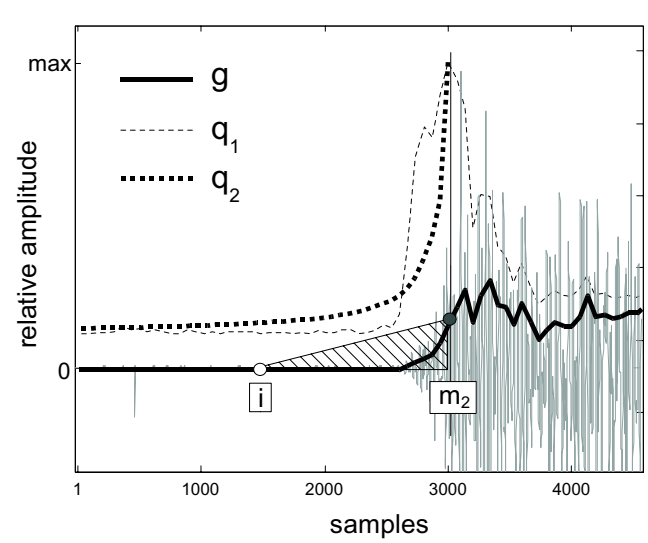

Fig.3: vector $q_{2}$ computing

Before function $q_{2}$ implementation, it is necessary specify highest local gravity centers value index $m_{2}$ in the range till distinct rise point $m_{1}$. Generally, these numbers $m_{1}$ and $m_{2}$ can differ.

$$
m_{1}=\underset{i}{\operatorname{argmax}}\left\{q_{1}(i)\right\}, m_{2}=\underset{i \leqslant m_{1}}{\operatorname{argmax}}\{g(i)\}
$$

Conversely, function $q_{2}$ compares the sum of surfaces below each local gravity center vector samples joins between points $i$ and $m_{2}$ with the surface under relevant interval outer samples join (see fig. 3). Such ratio rapidly increases at the point of signal amplitude elevation over noise background:

$$
q_{2}(i)=\frac{\sum_{j=i+1}^{m_{2}}(g(j-1)+g(j))}{\left(m_{2}-i\right)\left(g\left(m_{2}\right)+g(i)\right)} \quad \text { for } i=1, \ldots, m_{2}-1
$$

By reason of the noise characteristics estimate it is necessary to reflect initial emission event record samples, which do not interfere to effective signal, naturally. This noisy part can be specified by the point $z_{s}$, where the function $q_{2}$ crosses certain limit $p_{z}$. Actually, algorithm debugging showed that in incomplete beginning signal record case function $q_{2}$ starts with higher value than limit $p_{z}$. According to numerical experiments results, optimum $p_{z}$ value lies in range $0.2-0.3$ :

$$
z_{s}=\min \left\{i \mid q_{2}(i)>p_{z}\right\}
$$

In case of record noise part, also local gravity center vector values are almost constant, analogous to statistical signal characteristics. Hence, the signal sample location, where auxiliary vector $g$ lastly exceeds the noise characterizing level, can be considered as an approximate AE signal arrival. Such as threshold level $p_{s}$, the maximum local gravity center vector value into sample $z_{s}$ is taken:

$$
p_{s}=\max \left\{g(i) \mid i \leq z_{s}\right\}
$$


Approximate arrival $z_{a}$ is finally obtained by following thresholding:

$$
z_{a}=\max \left\{i \mid g(i)<p_{s}\right\}
$$

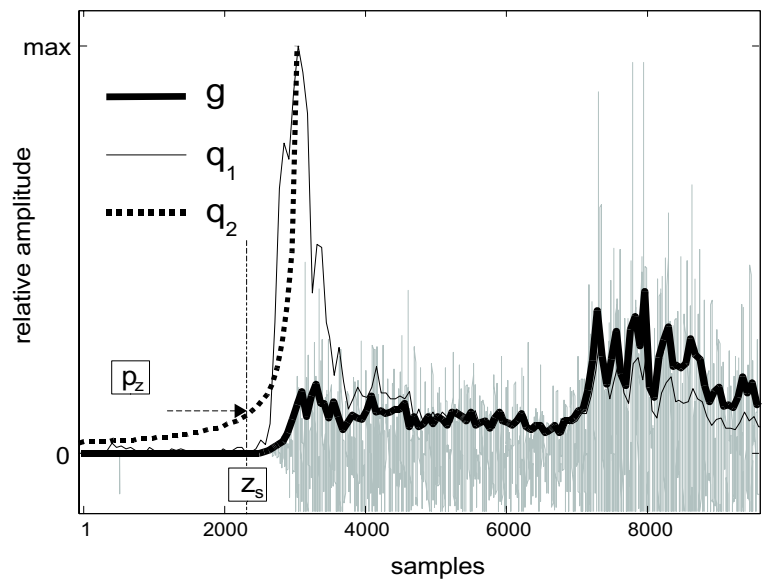

Fig.4: signal noise part determination

\subsection{Measuring window length factor}

Measuring window length is important accuracy affecting factor in relation to signal record noise level. In hypothetical ideal case, when the noise background is not present, immediate signal amplitude influences computed local gravity centers vector by $k$ samples earlier, then the currently evaluated element is. Conversely, if the signal beginning is hidden by noise, gravity centers vector change will approve more gradually. After many numerical experiments results, the measuring window length factor $f_{w}$ was established to compensate this effect and to correct the estimated signal beginning position with the linear reference to the ratio of noise level and local gravity centers vector maximum. The factor's signal - to-noise ratio sensitivity is adjusted by constant $c_{w}$ :

$$
f_{w}=c_{w} \cdot \frac{p_{s}}{\max \{g(i)\}}
$$

\subsection{Signal arrival estimation}

Signal record noise part is relatively exactly specified by approximate arrival $z_{a}$. In this way provided noisy segment and it's characteristics are exploited in final algorithm improvement steps. As the auxiliary threshold level $p_{1}$, the sum of relevant gravity centers vector part mean value and standard deviation is considered:

$$
p_{1}=\operatorname{mean}\left\{g(i) \mid i \leqslant z_{a}\right\}+\operatorname{std}\left\{g(i) \mid i \leqslant z_{a}\right\}
$$

The last vector $g(i)$ sample, that is less than value $p_{1}$, is rated as first signal onset estimate (see fig.5) and subsequently corrected by measuring window length factor:

$$
z_{1}=\max \left\{i \mid g(i)<p_{1}\right\}+k \cdot f_{w}
$$




\section{IVrd NDT in PROGRESS}

\subsection{Correction to zero crossing}

Each signal arrival estimate can be put more precisely by correction to zero crossing. It concern finding the last place before the actual arrival estimate location $z_{\text {act }}$, where the signal virtually comes through the mean value. The sample on the right from this place is taken as the new signal beginning version $z_{n}$ :

$$
z_{n}=\max \left\{i\left|i \leqslant z_{\text {act }} \wedge\right| d(i) \mid \neq 0\right\} \text {, where } d=\operatorname{diff}(\operatorname{sign}(s))
$$

All introduced signal arrival versions are always revised to "zero crossing".

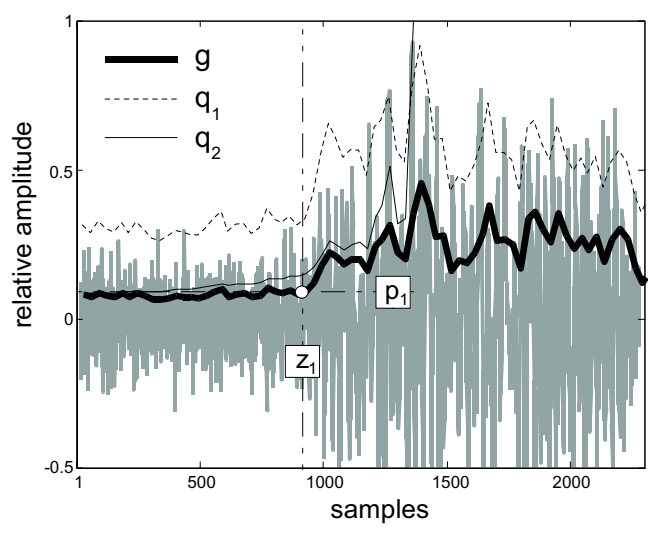

Fig.5: vector g(i) thresholding

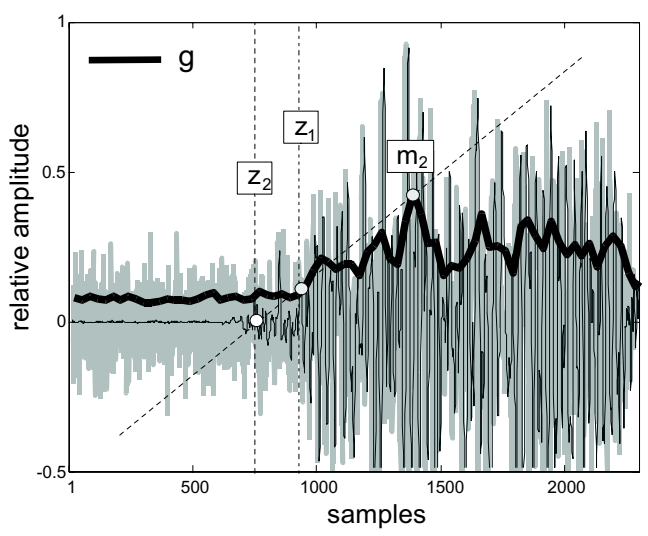

Fig.6: signal arrival linear extrapolation

\subsection{Linear extrapolation of signal arrival}

Particularly in cases of high signal noise levels, exact arrival estimate is more complicated. Using such oscilloscope data record, the signal onset recognition is not unique even by naked eye. This problem is illustratively shown on fig. 6 with typical record and the same one with high level white noise added. It is evident that original signal arrival is entirely covered by noise and the detection method lost it's exactness (see fig.6 - arrival version $z_{1}$ ). Hence, the signal arrival linear extrapolation was proposed. This elementary solution helps also in case of incomplete data record. Due to the general signal's linear initial amplitude rise, the first onset detection improvement can be done by the linear extrapolation marked on fig.6. The line comes through the points with coordinates $\left[m_{2}, g\left(m_{2}\right)\right]$ and $\left[z_{1}, g\left(z_{1}\right)\right]$. X-coordinate intersection $Z_{2}$ estimates the signal arrival more exactly.

Evidently, when the signal noise level is low, such arrival location extrapolation $z_{2}$ basically keeps the position $z_{1}$ unchanged. If the version $z_{1}$ is located near the record start, resulting value $z_{2}$ can be even negative. Thereby, certain not registered signal start is automatically restored. The benefits of this extrapolation were evidently proved by many numerical experiments.

\section{Other algorithm versions}

Regarding very similar features, certain method alternative can be represented by analogical local RMS values finding. All algorithm steps may be unchanged, except 
the auxiliary vector $g(i)$ (originally see 2.4 ) should be computed as the relevant signal part $s_{i}$ of RMS parameter:

$$
g_{R M S}(i)=R M S\left(s_{i}\right)=\sqrt{\frac{1}{k_{i}} \sum_{j=1}^{k_{i}}\left(s_{i}(j)\right)^{2}}
$$

Next algorithm steps, i.e. computing of functions $q_{1}$ and $q_{2}$, thresholding, and arrival estimate corrections, remain similar. However, unlike local gravity centers version, parameter RMS approved itself somewhat sensitive to immediate signal amplitude and this method modification looses robustness against various interferences in data record. Obversely, during numerical testing, RMS parameter proved better in higher noise level cases.

Within noise development observation by auxiliary vectors $g$ a $g_{R M S}$ it is possible to establish an alternative threshold values $p_{\text {alt, }}$ e.g. the noise part maximum or mean of both auxiliary vectors $g$ and $g_{R M S}$, including partial GC and RMS of relevant vectors $g$ and $g_{R M S}$ respectively:

$$
p_{\text {alt }}=\max / \text { mean/GC/RMS }\left\{g_{(R M S)}(i) \mid i \leqslant z_{a}\right\}
$$

\section{Parameters adjustment}

Algorithm parameter values optimization was made using the set of 150 randomly selected AE signals measured during complex aircraft structure part pen-test excitation. For the method precision examination, not only the original high signal - to - noise ratio records were used, but also similar ones with the white noise added. The estimative optimal parameter values were obtained by sequential approximation during numerical experiments without analytic computation. Each arrival estimation method has different proper parameter settings, naturally. Nevertheless, for given testing data set, the optimal values are in the neighbourhood of next ones:

$$
c_{k} \simeq 1.3, p_{z} \simeq 0.25, c_{w} \simeq 8
$$

With above mentioned adjustment, the new algorithm achieves significantly better results (the best mean absolute error was 18 samples) than edge detection method (mean absolute error was 199 samples). Considering appropriate sampling frequency $(200 \mathrm{~ns})$ and ultrasound pulse propagation velocity $(6.18 \mathrm{~mm} / \mathrm{us})$, we obtain resulting $A E$ event localization improvement from 246 to $103 \mathrm{~mm}$.

\section{Algorithm acceleration}

Whole signal length auxiliary vectors computing can be quite time - consuming and basically useless. For signal development casual information there is no need to calculate all samples of auxiliary vectors $g$ a $g_{R M S}$. However, it is fully sufficient to shift measuring window within half of it's length, thus, by $k$ samples. Such as resolution is enough for distinct rise $m_{2}$ estimative determination. Indeed, after that it is necessary to recalculate auxiliary vectors in full resolution, nevertheless, just from the point where vector $q_{2}$ exceeds the value about 0.21 (signal noise part mostly gradually ends here) to sample $m_{2}$. Considerable numerical operations amount is saved this way, so the algorithm is much faster and effectually applicable. 


\section{IVrd NDT in PROGRESS}

\section{Conclusions}

In contribution, the new $\mathrm{AE}$ signal arrival detection algorithm is described. Main features of the method can be summarized into following points:

- Emission event signal arrival is determined in terms of auxiliary local gravity centers and energy (RMS) vectors analysis.

- Suitably sampled signal is the unique algorithm input. An automatic measuring window width adjustment and signal record noise part estimation is implemented. Newly designed internal functions reliably separate the noise through whole it's approximate length, not only priorly set partition.

- New method is featured by robustness and reliability. It is debugged using real experiment extensive data set. The electrical interferences and $A D$ converter failures sensitivity is highly suppressed.

- Algorithm parameters were numerically optimized. The accuracy achieved is markedly better then that one of up to now applied methods.

- In incomplete signal record detection and higher noise level cases, signal arrival linear extrapolation is performed, whereby the noisy or missing signal initiation is reconstructed.

- Optimized algorithm implementation in Matlab provides sufficiently fast commonly processed $\mathrm{AE}$ signal records analysis.

\section{Acknowledgments}

The work was supported by the Grant Agency of the Czech Republic under project no. 101/07/1518 and by the Czech Ministry of Trade and Industry under project no. FT-TA/026-T9.

\section{References}

[1] Blahacek M.: Acoustic Emission Source Location Using Artificial Neural Networks. PhD. Thesis, Institute of Thermomechanics AS CR, Prague 2000 (in Czech).

[2] Petrik M., Kus V.: Localization of Acoustic Emission Sources by Means of Statistical Methods, $2^{\text {nd }}$ Internat. Workshop "NDT in Progress 2003", Prague, 6-8 Oct. 2003, Proc. ed. by P. Mazal, CNDT 2003, pp. 320. 